central protein in T4S functioning is the coupling protein, which belongs to the superfamily of SpoIIIE/FtsK proteins [10]. T7S is also dependent on a member of this protein family, which recognizes the C-terminal secretion signal [11]. In T6S systems, an ATPase of the AAA + family fulfils an essential, but not completely understood, role in assembly of different components of the secretion machinery [12]. Similarly, a member of the same family is also essential for T7S secretion. In line with these findings, we expect to find more functional similarities between T7S and especially $\mathrm{T} 4 \mathrm{~S}$ and T6S in the future.

Possibly, the confusion of Desvaux and colleagues [1] is due to the fact that they were focusing on T7S-like systems in the Firmicutes. Some of these monoderm bacteria (e.g. Staphylococcus aureus, Bacillus anthracis) have a functional T7S-like secretion system [13,14]. However, the similarity between these secretion systems and those in the mycolic-acid containing bacteria is very low. In fact, of the 5-7 conserved and essential components of the mycobacterial T7S systems only two are present in T7S systems of the Firmicutes [2]. Therefore, we have previously proposed to call the latter secretion systems 'type $7 \mathrm{~b}$ '. These secretion systems probably enable translocation across the cytoplasmic membrane, similar to the Sec and Tat systems, whereas the more extensive mycobacterial T7S systems mediate transport across both membranes and therefore perform 'true' secretion according to Desvaux and colleagues. This is, again, not without precedent because variants of T4S systems are also identified in conjugal plasmids from Gram-positive bacteria [15].
2 Abdallah, A.M. et al. Type VII secretion - mycobacteria show the way. Nat. Rev. Microbiol. 5, 883-891.

3 Brennan, P.J. (2003) Structure, function, and biogenesis of the cell wall of Mycobacterium tuberculosis. Tuberculosis (Edinb.) 83, 91-97

4 Sutcliffe, I.C. (1998) Cell envelope composition and organisation in the genus Rhodococcus. Antonie Van Leeuwenhoek 74, 49-58

5 Zuber, B. et al. (2008) Direct visualization of the outer membrane of mycobacteria and corynebacteria in their native state. J. Bacteriol. 190, $5672-5680$

6 Hoffmann, C. et al. (2008) Disclosure of the mycobacterial outer membrane: cryo-electron tomography and vitreous sections reveal the lipid bilayer structure. Proc. Natl. Acad. Sci. U. S. A. 105, 39633967

7 Niederweis, M. (2003) Mycobacterial porins-new channel proteins in unique outer membranes. Mol. Microbiol. 49, 1167-1177

8 Faller, M. et al. (2004) The structure of a mycobacterial outermembrane channel. Science 303, 1189-1192

9 Fortune, S.M. et al. (2005) Mutually dependent secretion of proteins required for mycobacterial virulence. Proc. Natl. Acad. Sci. U. S. A. 102, 10676-10681

10 Christie, P.J. et al. (2005) Biogenesis, architecture, and function of bacterial type IV secretion systems. Annu. Rev. Microbiol. 59, 451- 485

11 Champion, P.A. et al. (2006) C-terminal signal sequence promotes virulence factor secretion in Mycobacterium tuberculosis. Science $313,1632-1636$

12 Bonemann, G. et al. (2009) Remodelling of VipA/VipB tubules by ClpVmediated threading is crucial for type VI protein secretion. EMBO J. $28,315-325$

13 Burts, M.L. et al. (2005) EsxA and EsxB are secreted by an ESAT-6-like system that is required for the pathogenesis of Staphylococcus aureus infections. Proc. Natl. Acad. Sci. U. S. A. 102, 1169-1174

14 Garufi, G. et al. (2008) ESAT-6-like protein secretion in Bacillus anthracis. J. Bacteriol. 190, 7004-7011

15 Abajy, M.Y. et al. (2007) A type IV-secretion-like system is required for conjugative DNA transport of broad-host-range plasmid pIP501 in gram-positive bacteria. J. Bacteriol. 189, 2487-2496

\title{
References
}

1 Desvaux, M. et al. (2009) Secretion and subcellular localizations of bacterial proteins: a semantic awareness issue. Trends in microbiology

\section{Outer membrane translocation: numerical protein secretion nomenclature in question in mycobacteria}

\author{
Mickaël Desvaux ${ }^{1 *}$, Michel Hébraud ${ }^{1}$, Régine Talon ${ }^{1}$ and lan R. Henderson ${ }^{2}$ \\ ${ }^{1}$ INRA, UR454 Microbiologie, F-63122 Saint-Genès Champanelle, France \\ ${ }^{2}$ The University of Birmingham, Birmingham B15 2TT, United Kingdom
}

Consistent numerical classification of protein secretion systems according to the outer membrane translocation mechanisms is crucial in diderm bacteria. The mycobacterial ESAT-6 secretion system (Ess), more generically defined as the WXG100 secretion system (Wss), is clearly dedicated to protein translocation across the cytoplasmic membrane. Though, naming this system a "T7SS" is quite premature as the existence, nature and specificity of a putative outer membrane translocon that would enable

\footnotetext{
Corresponding author: Desvaux, M. (mickael.desvaux@clermont.inra.fr).

Institut National de la Recherche Agronomique (INRA), Centre de Recherche Clermont-Ferrand, UR454 Microbiologie, Site de Theix, F-63122 Saint-Genès Champanelle, France. Tel.: +33 (0)4 736247 23; fax: +33(0)4 73624581
}

true protein secretion in mycobacteria remains completely unknown.

In mycobacteria, the mycolic layer serves as a second lipid layer and contains well characterised $\beta$-barrel shaped or channel forming outer membrane proteins [1]. Based on these data, and in accordance with our previous article [2], Wilbert Bitter et al. [3] suggest that members of the high $\mathrm{G}+\mathrm{C} \%$ Gram-positive bacteria (Phylum BXIV Actinobacteria) sharing such features should be considered as diderm bacteria, i.e. bacteria with a two-membrane cell envelope. Furthermore, they argue that the use of the term "T7SS" to describe the Ess in mycobacteria is justified as these bacteria are didermata. 
While mycobacteria can be acknowledged as possessing two concentric biological membranes, the limit to the argument is the use of the term "T7SS" since, as already mentioned, there is a distinct absence of experimental evidence for protein translocation machinery within the outer membrane [2]. The presence of such additional secretion components is a prerequisite to incorporate a system within the established numerical classification for diderm bacteria [2]. Described as "possible" by the authors themselves, the presence of translocon components encoded by ESX-1 within the waxy outer sheath remains speculative; none has been identified, not even by genomic analyses. To date, ESX-1 has only been demonstrated as encoding translocon components described as, or predicted to be, localized within the cytoplasmic membrane and enabling protein translocation across it. As such, the Ess is at the same level of Sec, Tat and holin translocation systems, which are not included in the numerical protein secretion nomenclature [2,4]. Altogether, it seems premature to consider this system as a "T7SS" until further experimental evidence is provided to support the supposition that it mediates protein transport across both membranes in mycobacteria.

In the eventuality of such an outer membrane translocon being identified in mycobacteria, it would not necessarily be specific to the Ess as additional scenarios can be considered from what is known about protein secretion in other diderm bacteria [4] (Figure 1). It is possible that an outer membrane translocon is used by proteins exported in the first instance by different cytoplasmic membrane translocation systems, for example Sec- or Tat-exported proteins converge afterwards to the type II secretion system (T2SS). At the opposite, some proteins exported by a cytoplasmic membrane translocation system can be further translocated across the outer membrane by different systems, for example Sec-exported proteins are subsequently translocated across the outer membrane either by the T2SS, T4SS or T5SS. At the moment, it is not possible to settle on and ascertain one of these three possibilities for the Ess in mycobacteria. In addition to Ess, Sec and Tat are also present in mycobacteria and proteins translocated by these systems must further cross the outer membrane to be secreted into the extracellular milieu, but it remains completely unknown whether these exoproteins use a specific or a common outer membrane translocon. Naming the Ess a "T7SS" could be a mistake if it later appears this mycobacterial translocon, whenever it exists, is neither specific to the Ess nor specific to secreted proteins. It is noteworthy that none T2SS has been identified in mycobacteria despite the presence of Sec and Tat; attributing the "T7SS" designation to Ess is precipitate and could lead to the same confusion and mistakes when describing Sec as T2SS or T2SS as Gsp in the past [4]. Moreover, even though the cell envelope of mycobacteria is composed of two membranes, this outer membrane is quite unusual and not homologous to the outer membrane found in Gramnegative bacteria [1]. Nonetheless, the identification of such a non-homologous outer membrane translocon in mycobacteria could constitute an additional numerically classified protein secretion system, e.g. T9SS, as T7SS was proposed for the chaperone-usher pathway and T8SS for the extracellular nucleation-precipitation pathway [2].

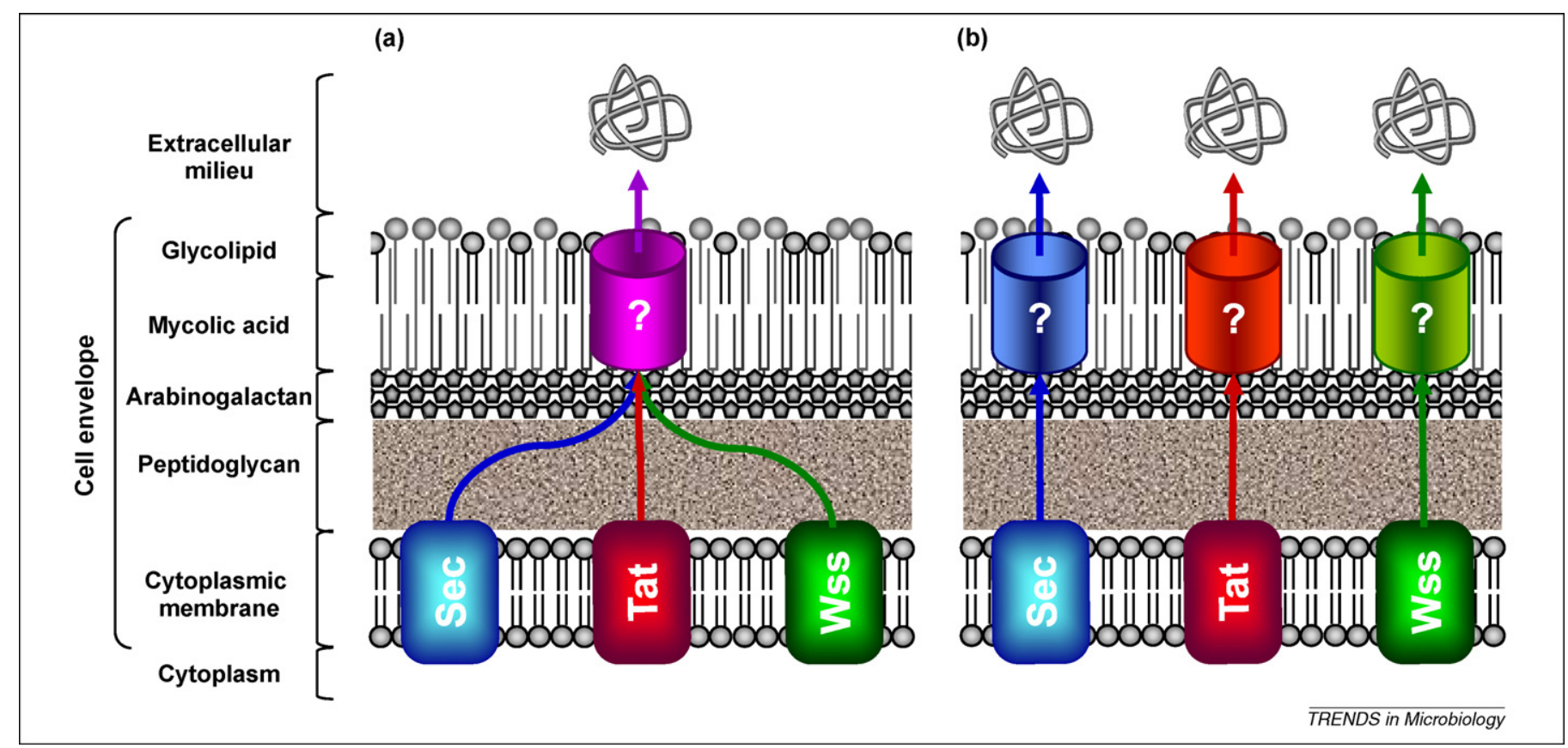

Figure 1. Hypothetical scenarios for protein secretion across the cell envelope of diderm mycobacteria. At least two extreme scenarios can be hypothesized in mycobacteria: proteins exported in the first instance across the cytoplasmic membrane via the Sec, Tat or Wss can be further truly secreted across the waxy outer sheath via (a) a common translocon, or (b) translocons specific to each of cytoplasmic-membrane translocons. Among alternative possibilities between these two extreme scenarios, proteins exported by a defined cytoplasmic-membrane translocation system and secreted by different translocons present in the waxy outer sheath can be mentioned, as observed in other diderm bacteria with Sec-exported proteins that can be further translocated across the outer membrane via T2SS, T4SS or T5SS, for example. As such translocons involved in protein translocation across the mycolic layer have not been identified so far in mycobacteria, it is currently not possible to ascertain whether or not they are homologous to other secretion systems described in other diderm bacteria such as the T2SS, or would constitute an alternative secretion system that could be named T9SS, for example. 
From our point of view, we would still refrain from naming this protein secretion system a "type $7 \mathrm{~b}$ " in monoderm bacteria, e.g. Staphylococcus aureus or Bacillus anthracis, as it could only reinforce confusion within the nomenclature. Indeed, and as agreed by Wilbert Bitter et al. themselves [3], (alpha-)numerical classification of protein secretion systems should only be applied to diderm bacteria. Naming it the WXG100 secretion system (Wss) clearly indicates it allows secretion of proteins belonging to the superfamily WXG100 (protein of about 100 amino acids long with a conserved WXG motif), which encompasses ESAT-6 (early secreted antigen of $6 \mathrm{kDa}$ ) and CFP-10 (culture filtrate protein of $10 \mathrm{kDa}$ ) from Mycobacterium tuberculosis [5]. If, in the end, this system encodes specific and additional outer membrane components in diderm mycobacteria, it could be called the T9SS and retain the Wss designation in monoderm bacteria, as is already the case for the T2SS and FPE or T3SS and FEA [2]. Concerning T4SS-like, it would be advisable to find another name to designate it in monoderm bacteria [6].

Unless a secretion system is perfectly characterised, it is difficult to classify it definitively. Our aim was not to establish an authoritative classification but to provide a reference point mentioning pitfalls and ambiguities in the nomenclature and to propose a starting point for coherent and logical solution to these issues, being as meticulously truthful as possible in order to suit a broad range of situations, such as secretion within mycobacteria. Tidying up the nomenclature is quite timely and necessary in the post-genomic era as global and integrative-biology approaches, namely genomics, transcriptomics and proteomics, rely on querying databases that need to be as clear and unambiguous as possible, not only to prevent promulgation of incorrect annotation but also to permit efficient cross interrogations. Our proposal for describing the protein secretion systems in bacteria will suffer the test of time and only the future will tell whether or not it has been sustained as our views and concepts need to be approved, adopted and further improved by the rest of scientific community within the field.

\section{References}

1 Song, H. et al. (2008) Identification of outer membrane proteins of Mycobacterium tuberculosis. Tuberculosis 88, 526-544

2 Desvaux, M. et al. (2009) Secretion and subcellular localizations of bacterial proteins: a semantic awareness issue. Trends Microbiol 17, 139-145

3 Bitter, W. et al. (2009) Type VII secretion in mycobacteria: classification in line with cell envelope structure. Trends Microbiol 17, 337-338

4 Desvaux, M. et al. (2004) The general secretory pathway: a general misnomer? Trends Microbiol 12, 306-309

5 Pallen, M.J. (2002) The ESAT-6/WXG100 superfamily- and a new Grampositive secretion system? Trends Microbiol 10, 209-212

6 Abajy, M.Y. et al. (2007) A type IV-secretion-like system is required for conjugative DNA transport of broad-host-range plasmid pIP501 in gram-positive bacteria. J Bacteriol 189, 2487-2496

0966-842X/\$ - see front matter ( $\odot 2009$ Published by Elsevier Ltd. doi:10.1016/j.tim.2009.05.008 Available online 11 August 2009 